\title{
RECENT RESULTS FROM CANGAROO
}

\author{
MASAKI MORI \\ FOR THE CANGAROO TEAM* \\ Institute for Cosmic Ray Research, University of Tokyo \\ 5-1-5 Kashiwanoha, Kashiwa, 277-8582 Chiba, Japan \\ E-mail: morim@icrr.u-tokyo.ac.jp
}

\begin{abstract}
The CANGAROO-III telescope system for very-high-energy gamma-ray astrophysics consists of four $10 \mathrm{~m}$ atmospheric Cherenkov telescopes located near Woomera, South Australia. The construction of the fourth telescope was completed in summer 2003, and stereoscopic observations has been in progress since March 2004. Here we report on the status of the system and some recent results from CANGAROO-III observations.
\end{abstract}

\section{Introduction}

CANGAROO is an acronym for the Collaboration of Australia and Nippon (Japan) for a GAmma Ray Observatory in the Outback. After successful operation of the $3.8 \mathrm{~m}$ imaging Cherenkov telescope (CANGAROO-I) for 7 years, which was the first of this kind in the southern hemisphere, we constructed a new telescope of $7 \mathrm{~m}$ diameter ${ }^{1}$ (CANGAROO-II) in 1999 next to the $3.8 \mathrm{~m}$ telescope near Woomera, South Australia $\left(136^{\circ} 47^{\prime} \mathrm{E}, 31^{\circ} 06^{\prime} \mathrm{E}\right.$, $160 \mathrm{~m}$ a.s.l.). Then the construction of an array of four $10 \mathrm{~m}$ telescopes (CANGAROO-III) was approved and as the first step the $7 \mathrm{~m}$ telescope was upgraded to $10 \mathrm{~m}$ diameter in 2000 , with this becoming the first telescope of the CANGAROO-III array ${ }^{2,3,4,5,6}$. Results from observations with this first $10 \mathrm{~m}$ telescope have been reported in publications (see, e.g. ${ }^{7}$ ).

In the following years, we have constructed an additional three $10 \mathrm{~m}$ telescopes located at the corners of a diamond of $100 \mathrm{~m}$ sides with improved mirrors, cameras and electronics. After tuning, we have started observation with the full system in stereo mode in March 2004 (Fig.1) ${ }^{8}$.

The major parameters of the CANGAROO-III telescopes are summarized in Table 1. The detailed design of reflectors ${ }^{9,10}$, cameras ${ }^{11,12,13}$,

*see http://icrhp9.icrr.u-tokyo.ac.jp for the full collaboration list. 


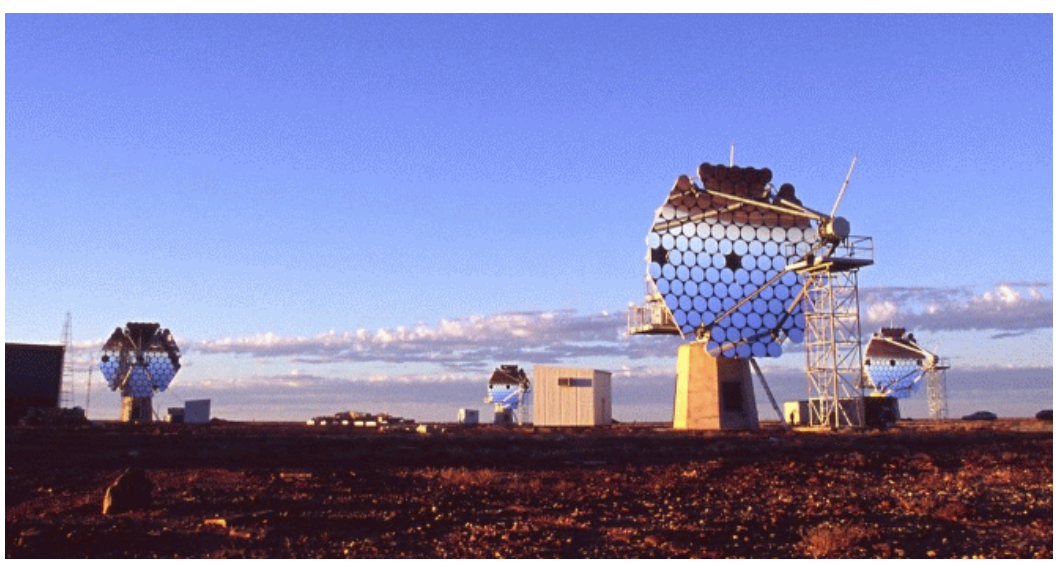

Figure 1. The CANGAROO-III telescopes in Woomera, South Australia, as of March 2004. From the left to right, they are called T2, T3, T4 and T1 in the order of construction. T1 was called CANGAROO-II before.

electronics ${ }^{14,15}$, and telescope control system ${ }^{16}$ are described elsewhere.

Table 1. Parameters of the CANGAROO-III telescopes.

\begin{tabular}{|l|c|c|}
\hline & T1 & T2, T3, T4 \\
\hline Mount & \multicolumn{2}{|c|}{ Alt-azimuth } \\
Focal length & \multicolumn{2}{|c|}{$8 \mathrm{~m}$} \\
Number of mirrors (area) & \multicolumn{2}{|c|}{$114\left(57 \mathrm{~m}^{2}\right.$ in total $)$} \\
Reflector type & \multicolumn{2}{|c|}{ Parabola } \\
Number of PMTs & $552\left(1 / 2^{\prime \prime}\right)$ & $427\left(3 / 4^{\prime \prime}\right)$ \\
Camera pixel size & $0.115^{\circ}$ & $0.168^{\circ}$ \\
Readout & TDC(CAMAC) \& ADC & TDC(VME) \& ADC \\
Point image size (FWHM) & $0.20^{\circ}$ & $0.14 \sim 0.21^{\circ}$ \\
Completion & 2000.3 & $2002.3(\mathrm{~T} 2), 2002.11(\mathrm{~T} 3)$, \\
& & $2003.7(\mathrm{~T} 4)$ \\
\hline
\end{tabular}

\section{Stereo analysis: the case of the Crab nebula}

The Crab nebula is an established TeV gamma-ray source and is used as a calibration source to check performance of a Cherenkov telescope. However, from Woomera, it can be observed only at large zenith angles $\left(>53^{\circ}\right)$. For stereo observations, the threshold energy of T1 is higher than other telescopes and thus we used the newer three telescopes for analysis. Because of the geometrical arrangement of the array, the effective baseline for large zenith angle observations becomes short which makes stereo reconstruction of images difficult. 
To overcome the unfortunate situation described above, we developed new analysis methods ${ }^{17}$. To avoid the increased uncertainty of the intersection points, we introduced a new parameter, "IP distance" $\left(D_{\mathrm{IP}}\right)$, which is defined as the distance between the intersection point and centroid of images. Then we searched best intersection points which minimizes the image widths and the difference between distance and $D_{\mathrm{IP}}$. This results in better angular resolution as seen in the $\theta^{2}$ distribution in Monte Carlo simulations, where $\theta$ is the space angle between the source direction and the reconstructed arrival direction: gamma-ray signals should be seen as a peak toward $\theta^{2}=0$, whose sharpness depends on the angular resolution and the angular extent of a gamma-ray source.

We observed the Crab nebula in December 2003 in so-called wobble mode, changing the pointing directions $\pm 0.5^{\circ}$ in declination from the target every 20 minutes. After basic data quality check, such as rejecting runs affected by clouds, a total of 890 minutes data were used for further analysis.

In addition to the conventional square cuts method using image parameters to enhance gamma-ray fractions, we applied two different analyses: the Likelihood method ${ }^{18,19}$ and the Fisher Discriminant method ${ }^{17,20}$. In the latter method, effectiveness of the parameters for the gamma-ray-like event selection is evaluated using the simulation, and we can optimize the weights of the parameters in estimating the probability of gamma-ray-like events. Finally we obtained the spectrum of the Crab nebula in the energy range from 2 to $20 \mathrm{TeV}^{20}$, which is consistent within the statistical and systematic errors with other measurements ${ }^{21,22}$ (Fig.2).

\section{Recent results}

\subsection{Pulsar PSR 1706-44}

A detection of a gamma-ray signal from PSR 1706-44, which was one of the EGRET-detected pulsars, was reported using the data acquired by CANGAROO-I $3.8 \mathrm{~m}$ telescope ${ }^{23}$. The Durham group also reported a detection with their Mark 6 telescope ${ }^{24}$. H.E.S.S., however, claimed no detection from that direction ${ }^{25}$. We observed this source for 27 hours $(\mathrm{ON})$ and 29 hours (OFF) with CANGAROO-III in May 2004. Preliminary analyses using T2 and T3 telescope pair did not show a peak in the $\theta^{2}$ distribution 26. The upper limit from this result is shown in Fig. 3, which is lower than the flux reported by CANGAROO-I. Further analysis is underway and the details will be reported elsewhere. 


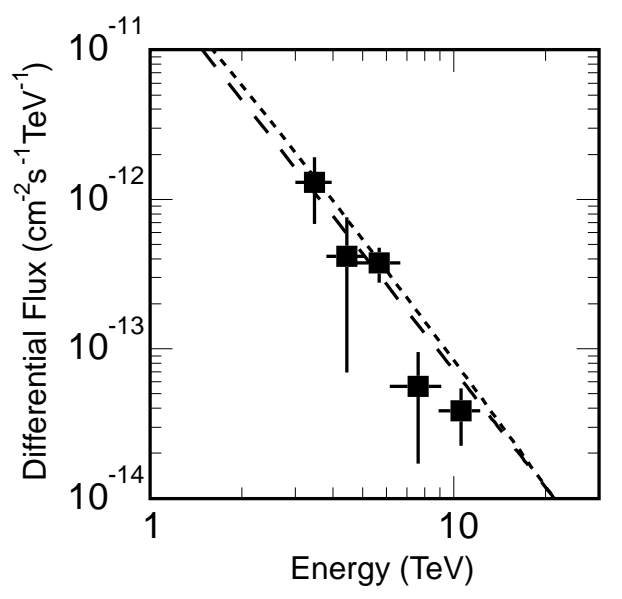

Figure 2. Differential gamma-ray flux from the Crab Nebula as a function of energy. The dashed line is the HEGRA result ${ }^{21}$ and the dotted one is the Whipple result ${ }^{22}$.

\subsection{Supernova remnant SN1006}

A detection of a gamma-ray signal from SN1006, which was shown to be a source of high-energy electrons through observation of non-thermal X-rays with $\mathrm{ASCA}^{27}$, was reported using the data acquired by CANGAROOI ${ }^{28}$. H.E.S.S., however, claimed no detection from that direction ${ }^{29}$. We observed this source for 27 hours (ON) and 29 hours (OFF) with CANGAROO-III in May 2004. Preliminary analyses using T2 and T3 telescope pair did not show any peaks in the $\theta^{2}$ distribution for the NErim point which was the maximum point of the gamma-ray emission in the CANGAROO-I data ${ }^{28}$. The upper limit from this result is lower than the flux reported by CANGAROO-I. Further analysis is underway to check the possible extended emission, and the details will be reported elsewhere.

\subsection{Vela pulsar and nebula}

The Vela pulsar was observed in January/February 2004. After basic data quality check, a total of 1311 minutes data were used for further analysis 20 , where the minimum elevation angle was set at $60^{\circ}$. The mean elevation angle was $70.9^{\circ}$, corresponding to an energy threshold of $600 \mathrm{GeV}$. The 


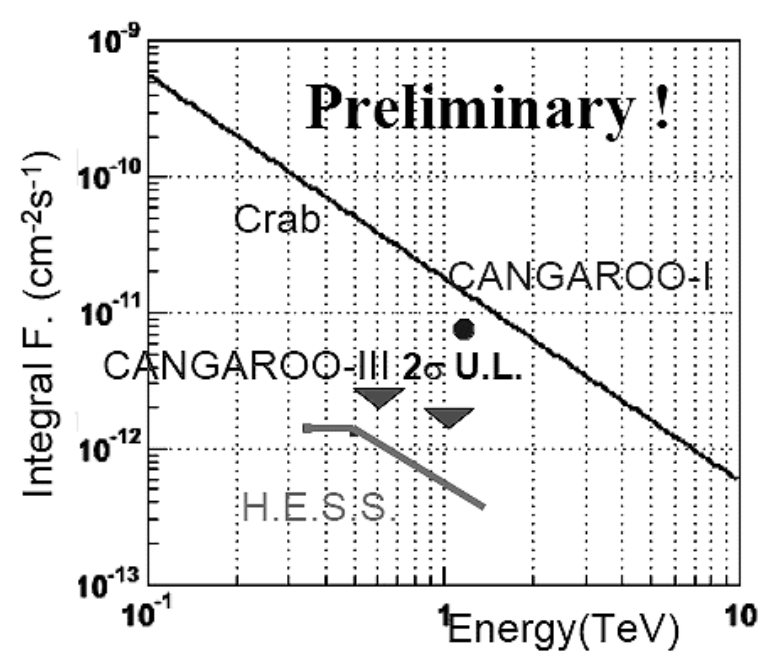

Figure 3. Upper limits on gamma-ray flux from PSR 1706-44 from CANGAROO-III observations (triangle) ${ }^{26}$. The CANGAROO-I result is shown by a filled circle ${ }^{23}$ and the H.E.S.S. limits ${ }^{25}$ are also shown.

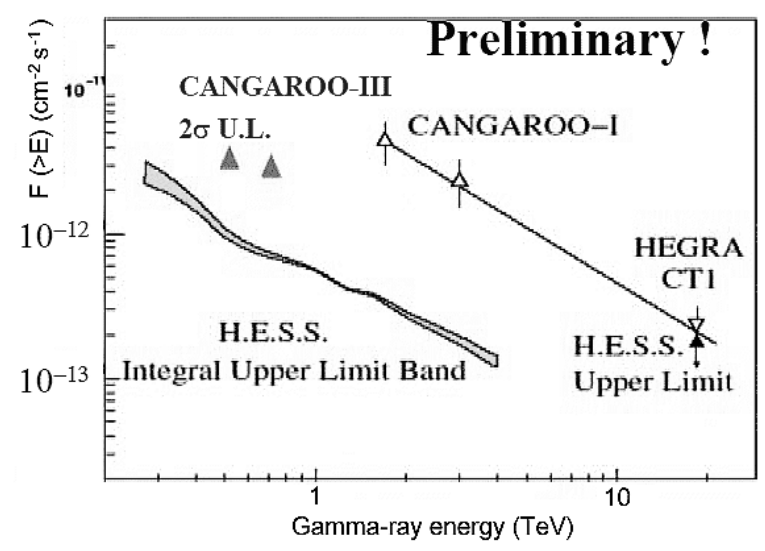

Figure 4. Upper limits on gamma-ray flux from NE-rim of SN1006. The CANGAROOI results are shown by open triangles ${ }^{28}$ and the HEGRA CT1 result by inverted open triangle ${ }^{30}$. The CANGAROO-III upper limits ${ }^{26}$ are shown by filled triangles with H.E.S.S. limits ${ }^{34}$

observations were carried out using the same wobble mode as for the Crab nebula observations. In this period, T2 and T3 were in operation, and we analyzed the stereo data from these two telescopes. For Vela, at a 
declination of $-45^{\circ}$, the relative orientation of the two telescopes does not present any problems.

We used the optimized analysis procedure used for the Crab nebula analysis described above. The resulting $\theta^{2}$ distribution for the Vela pulsar position showed no significant gamma-ray signal, giving upper limits as shown in Fig.5, which are consistent with H.E.S.S. results ${ }^{34}$. Also we did not see excess from the point offset by $0.13^{\circ}$ from the pulsar, which was the maximum of the excess detected with the CANGAROO-I telescope ${ }^{31}$.

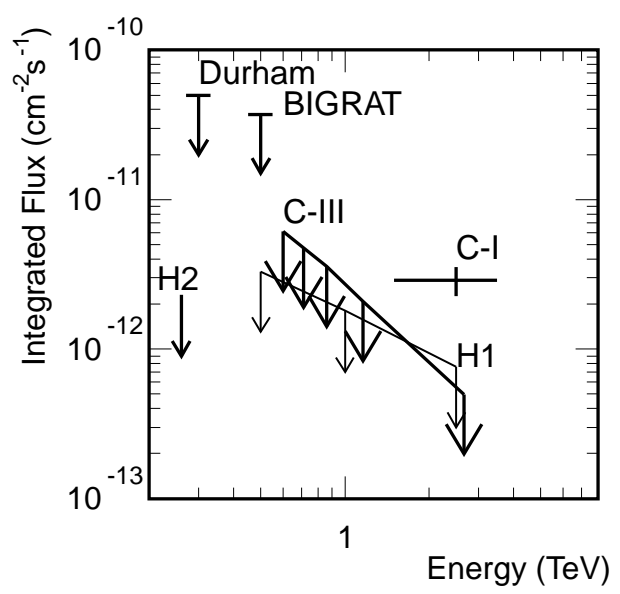

Figure 5. The $2 \sigma$ upper limits for the gamma-ray flux from the Vela pulsar by CANGAROO-III (C-III) ${ }^{20}$. C-I represents the CANGAROO-I excess from the point offset by $0.13^{\circ}$ from the pulsar ${ }^{31}$. Also shown are upper limits reported by the Durham group ${ }^{32}$, BIGRAT ${ }^{33}$ and H.E.S.S. ${ }^{34}$.

The H.E.S.S. group detected a gamma-ray excess from the Vela X nebula, extended over a $0.6^{\circ}$ radius from the center of the emission [(R.A., decl. $\left.)=\left(8^{\mathrm{h}} 35^{\mathrm{m}},-45^{\circ} 36^{\prime}\right)\right]^{34}$. In order to analyze extended emission, we applied the following method. Gamma-ray-like events can be extracted by fitting position-by position $F$ (Fischer discriminant) distributions under the assumption that gamma rays obey the Monte Carlo predictions, the proton background follows the average $F$ distribution of all directions, and the total distribution is a linear combination of those two. We chose the 
background region to be more than $0.8^{\circ}$ from the center, since we do not have sufficient statistics for off-source regions for these observations. The result of fitting is shown in Fig. 6. An excess was observed at $\theta^{2}<0.6 \mathrm{deg}^{2}$ around the center of the Vela $\mathrm{X}$ region. The excess radius is marginally consistent with H.E.S.S. considering our angular resolution. The total number of gamma-ray-like events is $561 \pm 114$. Though the statistical significance is below the $5 \sigma$ level, this could be a supporting evidence of the H.E.S.S. detection.

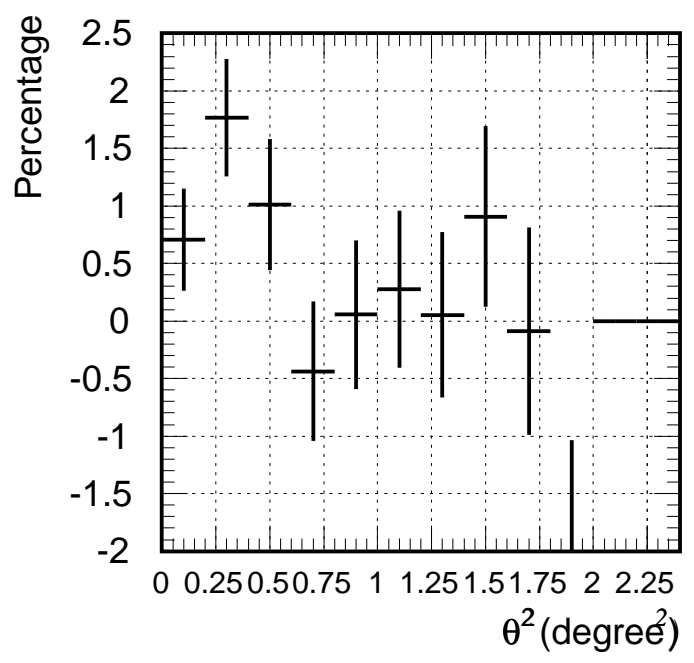

Figure 6. Wide-range $\theta^{2}$ plot for the Vela $\mathrm{X}$ region ${ }^{20}$, where $\theta$ is a space angle of an event direction from (R.A., decl.) $=\left(8^{\mathrm{h}} 35^{\mathrm{m}},-45^{\circ} 36^{\prime}\right)$, i.e., the peak of the emission detected by H.E.S.S. ${ }^{34}$.

The differential fluxes were obtained and compared with H.E.S.S. result in Fig. 7, which are in general agreement considering our poor statistics.

\subsection{SNR RX J0852.0-4622}

We reported a gamma-ray signal from this SNR using observations by CANGAROO-II ${ }^{35}$. We applied the Fisher discriminant method to the stereo data for RX J0852.0-4622 observed in January and February 2004 using T2 and T3 taken in the wobble mode for 2,197 minutes in total. We used the northwest rim as a target point in the wobble mode. After 


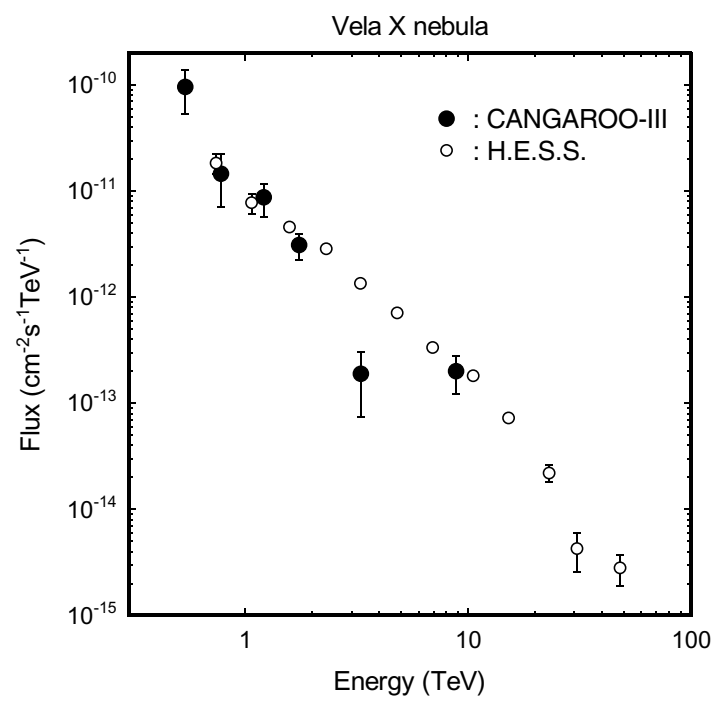

Figure 7. Gamma-ray spectra in the Vela X region observed by CANGAROO-III 20 compared with those reported by H.E.S.S. ${ }^{34}$.

the coarse selections, 1,204 minutes data were available. For the Fisher discriminant, we used four image parameters, lengths and widths, determined with each telescope independently. Finally the gamma-ray events were extracted by comparing the Fisher discriminant values between the SNR region and the background region. The excess count map is shown in Figure 8. Te region inside the solid arcs shows the maximum acceptance region, which is an overlap of the two field-of-views in the wobble mode. Also the one-degree arc from the SNR center is indicated by the dotted line. The strong gamma-ray emission from the NW rim is obviously seen, which was first reported by CANGAROO-II. This maximum acceptance region covers about a half of the whole SNR, and the integrated flux above $0.81 \mathrm{TeV}$ is about $60 \%$ of the H.E.S.S. result ${ }^{36}$, which value is reasonable considering our coverage of the SNR ${ }^{26,37}$.

\section{Summary}

We have been carrying out stereo observations of sub- $\mathrm{TeV}$ gamma-rays with CANGAROO-III since March 2004. Results from stereo observations were presented: PSR 1706-44 and SN1006, from which gamma-ray signals were reported by CANGAROO-I, were not confirmed by CANGAROO-III 


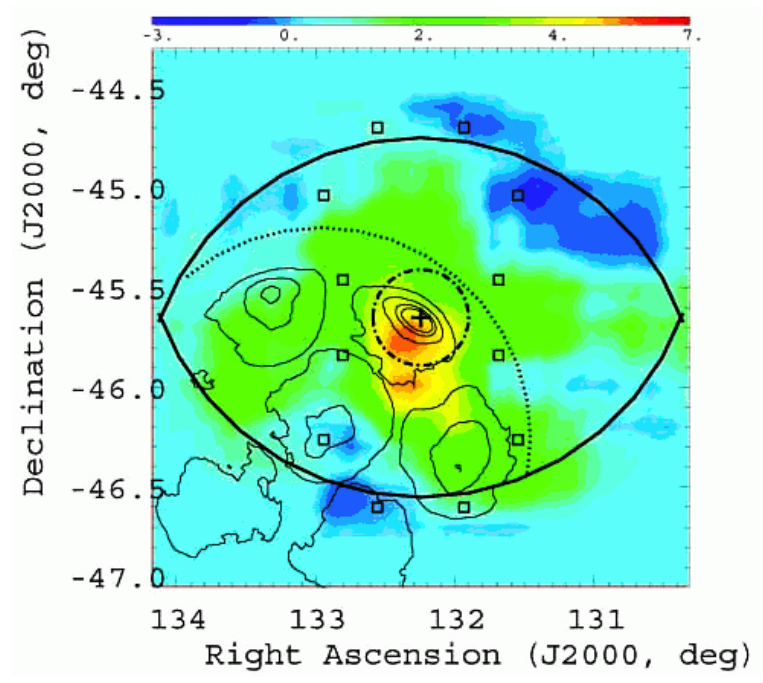

Figure 8. Excess event map around the SNR RX J0852.0-4622 obtained from the CANGAROO-III stereo observations in 2004 (preliminary) ${ }^{37}$.

observations. For two supernova remnants, the Vela SNR and RX J0852.04622 , our results are consistent with the recent H.E.S.S. results.

The distribution of 'gamma-ray SNRs' is important in the quest for the origin of cosmic-rays and the high-energy content of the Universe: we will continue systematic study of SNRs in high-energy gamma-rays in the Galaxy.

\section{References}

1. T. Tanimori et al., in Proc. 26th ICRC (Salt Lake City) (University of Utah, Utah), 5, 203-206 (1999).

2. M. Mori et al., in "GeV-TeV Gamma Ray Astrophysics Workshop: Towards a Major Atmospheric Cherenkov Detector V", (eds. B.L.Dingus, M.H.Salamon and D.B.Kieda, AIP Proceedings, New York, 2000), pp. 485-491.

3. T. Tanimori et al., in "Very High Energy Phenomea in the Universe", eds. M.Boer and J. Tran Than Van, GIOI Publishers, France, 2001), pp.105-108.

4. M. Mori et al., in Proc. 27th ICRC (Hamburg) (Copernicus Gesellshaft, Berlin, 2001), pp. 2831-2834.

5. R. Enomoto et al., in Proc. 28th ICRC (Tsukuba) (Universal Academy Press, Tokyo, 2003), pp. 2807-2810.

6. H. Kubo et al., New Astronomy Reviews 48, 323-329 (2004).

7. M. Mori et al., in AIP Conference Proceedings 745 (eds. F.A. Aharonian, H.J.Völk and D. Horns, AIP, New York, 2005), pp. 639-644. 
8. K. Nishijima et al., in Proc. 29th ICRC (Pune) (Tata Inst. Fundamental Research, India, 2005), Vol. 5, pp. 327-330.

9. A. Kawachi et al., Astropart. Phys. 14, 261-269 (2001).

10. M. Ohishi et al., in Proc. 28th ICRC (Tsukuba) (Universal Academy Press, Tokyo, 2003), pp. 2855-2858.

11. F. Kajino et al., in Proc. 27th ICRC (Hamburg) (Copernicus Gesellshaft, Berlin, 2001), pp. 2909.

12. S. Kabuki et al., in Proc. 28th ICRC (Tsukuba) (Universal Academy Press, Tokyo, 2003), pp. 2859-2862.

13. S. Kabuki et al., Nucl. Instr. Meth., A500, 318-336, 2003.

14. H. Kubo et al., in Proc. 27th ICRC (Hamburg) (Copernicus Gesellshaft, Berlin, 2001), pp. 2900-2903.

15. H. Kubo et al., in Proc. 28th ICRC (Tsukuba) (Universal Academy Press, Tokyo, 2003), pp. 2863-2866.

16. S. Hayashi et al., in Proc. 28th ICRC (Tsukuba) (Universal Academy Press, Tokyo, 2003), pp. 2867-2870.

17. T. Nakamori et al., in Proc. 29th ICRC (Pune) (Tata Inst. Fundamental Research, India, 2005), Vol. 4, pp. 203-206.

18. R. Enomoto et al., in Proc. 27th ICRC (Hamburg) (Copernicus Gesellshaft, Germany), Vol.5, pp. 2477-2480 (2001).

19. R. Enomoto et al., Nature 416, 823-826 (2002).

20. R. Enomoto et al., Astrophys. J. 638, 397-408 (2006).

21. F.A. Aharonian et al., Astrophys. J. 539, 317-324 (2000).

22. A.M. Hillas et al., Astrophys. J. 503, 744-759 (1998).

23. T. Kifune et al., Astrophys. J. 438, L91-94 (1995).

24. P.M. Chadwick et al., Astropart. Phys. 9, 131-136 (1998).

25. F.A. Aharonian et al., Astron. Astrophys. 432, L9-L12 (2005).

26. T. Tanimori et al., in Proc. 29th ICRC (Pune) (Tata Inst. Fundamental Research, India, 2005), Vol. 4, pp. 215-218.

27. K. Koyama et al., Nature, 278, 255-228 (1995).

28. T. Tanimori et al., Astrophys. J. 497, L25-28 (1998).

29. F.A. Aharonian et al., Astron. Astrophys. 437, 135-139 (2005).

30. V. Vitale et al., in Proc. 28th ICRC (Tsukuba) (Universal Academy Press, Tokyo, 2003), pp. 2889-2892.

31. T. Yoshikoshi et al., Astrophys. J. 487, L65-68 (1997).

32. P.M. Chadwich et al., Astrophys. J. 537, 414-421 (2000).

33. S.A. Dazeley, Ph.D. thesis, University of Adelaide (1999).

34. F.A. Aharonian et al., Astron. Astrophys. 448, L43-47 (2006).

35. H. Katagiri et al., Astrophys. J. 619, L163-L165 (2005).

36. F. Aharonian et al., Astron. Astrophys. 437, L7-10 (2005).

37. R. Enomoto et al., to be submitted for publication (2006). 